\title{
OPEN Metabolic factors and the risk of Dupuytren's disease: data from 30,000 individuals followed for over 20 years
}

Mattias Rydberg 11,2凶 Malin Zimmerman ${ }^{1,2}$ Jin Persson Löfgren ${ }^{1,2}$ Anders Gottsäter ${ }^{3}$ Peter M. Nilsson ${ }^{4,5}$, Olle Melander ${ }^{4,5}$ \& Lars B. Dahlin ${ }^{1,2}$

Dupuytren's disease (DD) is a fibroproliferative disorder affecting the palmar fascia of the hand. Risk factors include diabetes mellitus (DM), whereas a high body mass index (BMI) is associated with a lower prevalence of DD. The aim of this study was to further elucidate risk and protective factors for the development of DD using longitudinal population-based data from the Malmö Diet and Cancer Study (MDCS). During 1991-1996, the inhabitants aged 46-73 years in the city of Malmö, Sweden were invited to participate in the population-based MDCS (41\% participation rate). Data on incident DD were retrieved from Swedish national registers. Associations between DM, alcohol consumption, BMI, and serum apolipoprotein A1 (ApoA1) and apolipoprotein B (ApoB) at baseline were analysed in multivariable Cox regression models adjusted for known confounders. Among 30,446 recruited participants, 347 men and 194 women were diagnosed with DD during a median follow-up time of 23 years. DM (men HR 2.23; 95\% Cl 1.50-3.30, women HR 2.69; 95\% Cl 1.48-4.90) and alcohol consumption (men $\mathrm{HR} 2.46 ; 95 \% \mathrm{Cl} 1.85-3.27$, women $\mathrm{HR} 3.56 ; 95 \% \mathrm{Cl} 1.95-6.50$ ) were independently associated with incident DD in the Cox regression models. Furthermore, inverse associations with incident DD were found for obesity among men, and ApoB/ApoA1 ratio among both sexes. DM and excess alcohol consumption constituted major risk factors for the development of DD. Furthermore, an inverse association between obesity among men and DD, and also between ApoB/ApoA1 ratio and DD was found in both sexes.

Dupuytren's disease (DD) is a fibroproliferative disorder affecting the palmar fascia of the hand. It often starts with nodules in the hand that over time thickens and form cords, contracting the finger and thus causing Dupuytren's Contracture. Although any finger can be affected, DD is most often diagnosed in the ring and middle fingers ${ }^{1}$. The prevalence of DD varies significantly in the literature, depending on the region and population studied $^{2,3}$. A recent Swedish large-scale population-study found a 1.35\% prevalence of DD among men and $0.5 \%$ among women ${ }^{4}$. Treatment options include pharmacological injection of collagenase, needle fasciotomy, and open fasciectomy ${ }^{1}$.

The exact aetiology and pathogenesis of DD is still debated although several risk factors have been proposed, including smoking ${ }^{5}$, alcohol consumption ${ }^{6}$, hypertension ${ }^{7}$, manual work ${ }^{8}$, genetic susceptibility ${ }^{9}$, and diabetes mellitus $(\mathrm{DM})^{10}$. Moreover, different body anthropometrics, including body mass index (BMI), have been investigated in relation with DD with relatively consistent results, indicating lower risk for DD with higher BMI ${ }^{11,12}$. Interestingly, recent genetic studies have proposed a negative correlation between gene variants for increased $\mathrm{BMI}$ and $\mathrm{DD}$, supporting an inverse association between $\mathrm{BMI}$ and $\mathrm{DD}^{13}$. Finally, previous studies have associated DD with increased serum cholesterol and triglyceride levels ${ }^{14}$ and also with alterations in lipid deposition in the palmar fat tissue ${ }^{15}$. However, the number of longitudinal cohort studies in the DD field in general is low ${ }^{16}$, and studies aiming to control for potential confounding factors are lacking. Therefore, the aim of this observational study was to use longitudinal data from a population-based cohort study, the Malmö Diet and Cancer study

\footnotetext{
${ }^{1}$ Department of Hand Surgery, Lund University, Skåne University Hospital, Jan Waldenströms gata 5, 20502 Malmö, Sweden. 'Department of Translational Medicine-Hand Surgery, Lund University, Lund, Sweden. ${ }^{3}$ Department of Vascular Diseases, Lund University, Skåne University Hospital, Malmö, Sweden. ${ }^{4}$ Department of Emergency and Internal Medicine, Skåne University Hospital, Malmö, Sweden. ${ }^{5}$ Department of Clinical Sciences, Lund University, Malmö, Sweden. ${ }^{\varpi}$ email: mattias.rydberg@med.lu.se
} 


\begin{tabular}{|l|l|l|}
\hline Characteristics & Women $(\mathbf{n}=\mathbf{1 8 , 3 1 8})$ & Men $(\mathbf{n}=\mathbf{1 2 , 0 7 8})$ \\
\hline Age, years $($ mean \pm SD) & $56.8 \pm 7.9$ & $58.7 \pm 7.0$ \\
\hline BMI $\left(\mathbf{k g} / \mathbf{m}^{2}\right)(\mathbf{m e a n} \pm$ SD) & $25.5 \pm 4.3$ & $26.3 \pm 3.5$ \\
\hline Normal weight (n [\%]) & $9591(52.5)$ & $4488(37.2)$ \\
\hline Overweight (n [\%]) & $6088(33.3)$ & $5932(49.2)$ \\
\hline Obesity (n [\%]) & $2606(14.3)$ & $1633(13.5)$ \\
\hline Current smoking (n [\%]) & $4858(28.1)$ & $3218(28.7)$ \\
\hline Hypertension (n [\%]) & $7712(42.2)$ & $6437(53.4)$ \\
\hline Low alcohol consumption (n [\%]) & $14,303(83.7)$ & $6661(60)$ \\
\hline Moderate alcohol consumption (n [\%]) & $2388(14.0)$ & $2850(25.7)$ \\
\hline Heavy alcohol consumption (n [\%]) & $402(2.4)$ & $1585(14.3)$ \\
\hline Diabetes mellitus (n [\%]) & $656(3.6)$ & $742(6.1)$ \\
\hline Manual work (n [\%]) & $6594(38.5)$ & $4054(36.3)$ \\
\hline Apolipoprotein Al, g/L (mean \pm SD) & $1.64 \pm 0.3$ & $1.45 \pm 0.2$ \\
\hline Apolipoprotein B, g/L (mean \pm SD) & $1.05 \pm 0.3$ & $1.11 \pm 0.3$ \\
\hline Incident DD (n [\%]) & $194(1.1)$ & $347(2.9)$ \\
\hline Incidence rate DD (cases/1000 person-years) & 0.49 & 1.47 \\
\hline
\end{tabular}

Table 1. Baseline characteristics stratified for sex. Normal weight; $\mathrm{BMI}<25$, overweight; $\mathrm{BMI} \geq 25$ to $<30$, obese $\mathrm{BMI} \geq 30 \mathrm{~kg} / \mathrm{m}^{2}$. Low alcohol consumption $<15 \mathrm{~g} /$ day, Moderate alcohol consumption 15-30 g/day, Heavy alcohol consumption $>30 \mathrm{~g} /$ day. Participants with prevalent DD at baseline $(\mathrm{n}=50)$ were excluded. $D D$ Dupuytren's disease, $g$ grams, $S D$ standard deviation.

$(\mathrm{MDCS})^{17}$, in order to explore associations between DD and baseline DM, BMI, alcohol consumption, and apolipoprotein levels during long-term follow-up.

\section{Results}

Baseline characteristics. Baseline characteristics, stratified by sex, are presented in Table 1. Baseline characteristics stratified by incident DD and sex are presented in Table 2a,b. Fifty participants were excluded from further analysis due to a prevalent diagnosis of DD at baseline (Fig. 1). In total, there were 541 participants diagnosed with DD during a median follow-up of 23 [IQR 15.8-30.2] years; 347 men and 194 women. This corresponds to an incidence of 1.47 cases per 1000-person years for men, and 0.49 cases per 1000 person-years for women. Mean age at diagnosis was 71 years for both men (SD 7.0) and women (SD 7.5).

Incident DD by diabetes status, BMI and alcohol consumption. In total, there were 656 female and 742 male participants with prevalent DM at baseline (Table 1).

In the first sex-stratified Cox regression model, including only age and respective variable, prevalent DM at baseline was independently associated with DD among both men (HR 2.03; 95\% CI 1.38-3.00; p < 0.001) and women (HR 2.38; 95\% CI 1.32-4.29; $\mathrm{p}=0.004)$. When including all variables, i.e. age, BMI-group, alcohol consumption, smoking status, manual work and hypertension, in the multivariable Cox regression model, prevalent DM was still independently associated with incident DD among both men (HR 2.23; 95\% CI 1.50-3.30; $<<0.001)$ and women (HR 2.69; 95\% CI 1.48-4.90). Furthermore, among both men and women, there was a significant association between both moderate and heavy alcohol consumption and incident DD in both Cox regression models. Finally, in the multivariable model, there was an inverse association between obesity and incident DD among men (HR 0.66; 95\% CI 0.44-0.98 p =0.04). Among women, there was a borderline significant inverse association between obesity and DD (HR 0.55; 95\% CI 0.33-1.00 p = 0.05) (Table 3). No significant associations were found between smoking, hypertension, overweight, and incident DD, neither among men nor women.

Sensitivity analysis and interaction analysis. In the sensitivity analyses, the first excluding 1303 participants with a $\mathrm{BMI}<20.0$ and the second excluding 7956 participants with a follow-up time $>25$ years in the multivariable Cox regression model, the associations found between DM, alcohol consumption, obesity among men and DD remained unchanged (Supplementary Tables 1 and 2). No significant interactions were found between DM and BMI ( $p$ for interaction; men $p=0.42$, women $p=0.82$ ) or DM and alcohol consumption ( $p$ for interaction; men $\mathrm{p}=0.59$, women $\mathrm{p}=0.73$ ).

Finally, the associations between DM and DD remained unchanged when analyzing separate HRs for low and moderate to high alcohol consumers among both men and women (data not shown).

Incident DD by apolipoprotein levels. Table 2a,b display sex-stratified distributions of ApoA1 and ApoB, respectively. ApoA1 was higher at baseline in participants with incident DD. ApoB among women and the ApoB/ApoAl ratio among both men and women were lower in participants with incident DD.

In the fully adjusted Cox regression model, one SD increment of ApoA1 was associated with DD in both men $\left(\mathrm{HR}_{\text {per SD }} 1.22 ; 95 \% \mathrm{CI} 1.09-1.38 ; \mathrm{p}<0.01\right)$ and women $\left(\mathrm{HR}_{\mathrm{per} \mathrm{SD}} 1.17 ; 95 \% \mathrm{CI} 1.01-1.36 ; \mathrm{p}=0.03\right)$. ApoB was 


\begin{tabular}{|c|c|c|c|}
\hline (a) Male participants & $\begin{array}{l}\text { No DD } \\
n=11,731\end{array}$ & $\begin{array}{l}\text { Incident DD } \\
\mathbf{n}=347\end{array}$ & p value \\
\hline Age, years $($ mean $\pm S D)$ & $58.6 \pm 7.0$ & $56.8 \pm 6.4$ & $<0.001$ \\
\hline $\mathrm{BMI}\left(\mathrm{kg} / \mathrm{m}^{2}\right)($ mean $\pm \mathrm{SD})$ & $26.3 \pm 3.6$ & $25.9 \pm 3.3$ & $<0.028$ \\
\hline Current smoking (n [\%]) & $3118(28.7)$ & $100(30.5)$ & $=0.47$ \\
\hline Hypertension (n [\%]) & $6275(53.6)$ & $162(46.7)$ & $=0.01$ \\
\hline Diabetes mellitus (n [\%]) & $713(6.1)$ & $29(8.4)$ & $=0.08$ \\
\hline Alcohol consumption g/day [IQR] & $11.2[2.0-20.3]$ & $17.2[6.9-27.5]$ & $<0.001$ \\
\hline Manual work (n [\%]) & $3942(36.4)$ & $112(34.3)$ & $=0.43$ \\
\hline ApoA1, g/L $($ mean $\pm S D)$ & $1.45 \pm 0.2$ & $1.52 \pm 0.3$ & $<0.001$ \\
\hline ApoB g/L $($ mean \pm SD $)$ & $1.11 \pm 0.3$ & $1.10 \pm 0.3$ & $=0.5$ \\
\hline ApoB/ApoA1 ratio $($ mean $\pm S D)$ & $0.78 \pm 0.2$ & $0.74 \pm 0.2$ & $<0.001$ \\
\hline (b) Female participants & $\begin{array}{l}\text { No DD } \\
n=18,124\end{array}$ & $\begin{array}{l}\text { Incident DD } \\
n=194\end{array}$ & p value \\
\hline Age, years $($ mean $\pm S D)$ & $56.8 \pm 7.9$ & $56.1 \pm 7.0$ & $=0.18$ \\
\hline BMI $\left(\mathrm{kg} / \mathrm{m}^{2}\right)($ mean $\pm \mathrm{SD})$ & $25.5 \pm 4.3$ & $25.2 \pm 3.9$ & $=0.26$ \\
\hline Current smoking (n [\%]) & $4811(28.1)$ & $47(25.3)$ & $=0.4$ \\
\hline Hypertension (n [\%]) & $7644(42.3)$ & $68(35.2)$ & $=0.049$ \\
\hline Diabetes mellitus (n [\%]) & $643(3.5)$ & $13(6.7)$ & $=0.019$ \\
\hline Alcohol consumption g/day [IQR] & $5.4[0.1-10.7]$ & $6.9[0.2-13.6]$ & $=0.01$ \\
\hline Manual work (n [\%]) & $6523(38.5)$ & $71(38.6)$ & $=0.97$ \\
\hline ApoA1, g/L $($ mean $\pm S D)$ & $1.64 \pm 0.3$ & $1.71 \pm 0.3$ & $=0.01$ \\
\hline ApoB g/L (mean \pm SD) & $1.05 \pm 0.3$ & $1.00 \pm 0.2$ & $=0.01$ \\
\hline ApoB $/ A p o A 1$ ratio $($ mean $\pm S D)$ & $0.66 \pm 0.2$ & $0.61 \pm 0.2$ & $=0.003$ \\
\hline
\end{tabular}

Table 2. a, b Baseline characteristics with all subjects, stratified with regard to incident DD. Participants with prevalent $\mathrm{DD}$ at baseline $(\mathrm{n}=50)$ were excluded. ${ }^{\star} \mathrm{P}$-value for group comparison between subjects without $\mathrm{DD}$ and with incident DD; independent sample t-test used for age, BMI and lipid levels, Chi-Square test for dichotomous variables and Mann-Whitney $U$ test was used for alcohol consumption. Participants with prevalent DD are excluded. Bold values indicating $p<0.05$. APO Apolipoprotein, BMI body mass index, $D D$ Dupuytren's disease, $I Q R$ interquartile range, $S D$ standard deviation.

inversely associated with DD among women $\left(\mathrm{HR}_{\mathrm{per} \mathrm{SD}} 0.83 ; 95 \% \mathrm{CI} 0.70-0.99 ; \mathrm{p}=0.03\right)$, however no significant association was found among men. Finally, the ApoB/ApoA1 ratio was inversely associated with incident DD in both men $\left(\mathrm{HR}_{\text {per SD }} 0.85 ; 95 \%\right.$ CI $\left.0.75-0.96 ; \mathrm{p}=0.01\right)$ and women $\left(\mathrm{HR}_{\text {per SD }} 0.80 ; 95 \% \mathrm{CI} 0.66-0.97 ; \mathrm{p}=0.02\right)$ (Table 4).

\section{Discussion}

The findings from this longitudinal population-based study with a median follow up of 23 years corroborate that $\mathrm{DM}$ and excessive alcohol consumption constitute major risk factors for the development of DD. Furthermore, we found an inverse association between obesity among men and DD. We also present data proposing an inverse association between $\mathrm{ApoB} / \mathrm{ApoA} 1$ ratio and DD, findings that has not been previously reported. In addition to information regarding risk factors for $\mathrm{DD}$, the study sheds light on the complex associations between DD, DM, and high BMI.

Diabetes Mellitus. DM has long been considered a risk factor for DD, although the exact mechanisms by which hyperglycaemia affects the palmar fascia are still not fully understood ${ }^{1,10}$. One of the proposed biochemical processes underlying diabetic complications is the formation of advanced glycated end products (AGEs) ${ }^{18}$. AGEs have been associated with other fibroproliferative disorders, such as diabetic cardiomyopathy ${ }^{19}$ and idiopathic pulmonary fibrosis ${ }^{20}$, but also to complications of diabetes in the hand, e.g. carpal tunnel syndrome ${ }^{21}$. Indeed, data from biopsies from the palmar fascia in patients with DD showed higher levels of AGEs compared to a control group ${ }^{22}$, possibly increasing levels of collagen deposition and increased collagen stiffness ${ }^{10,22}$. Furthermore, previous studies have shown that DM increases the formation of myofibroblasts ${ }^{23}$, one of the proposed main cell types responsible for contraction the finger in $\mathrm{DD}^{24}$. Thus, future studies should examine the palmar fascia in patients with DM to clarify the effects of hyperglycaemia and AGEs on the development of DD. In our study, participants with prevalent DM at baseline had marked increased risk for development of DD during follow-up; even when adjusting for other known risk factors, e.g. alcohol consumption and BMI. Our results are in line with a previous meta-analysis on the effect of $\mathrm{DM}$ on the development of $\mathrm{DD}^{10}$. Our study adds important information due to its longitudinal setting and long follow-up, why it contributes to the establishment of DM as a major risk factor for the development of DD. Finally, our findings support the theory of DD as being a part of the more complex syndrome- "the diabetic hand", together with diagnoses, such as carpal tunnel syndrome, trigger finger, and ulnar neuropathy ${ }^{25-27}$. 


\section{Malmö Diet and Cancer Study} baseline 1991-1996

$n=30,446$

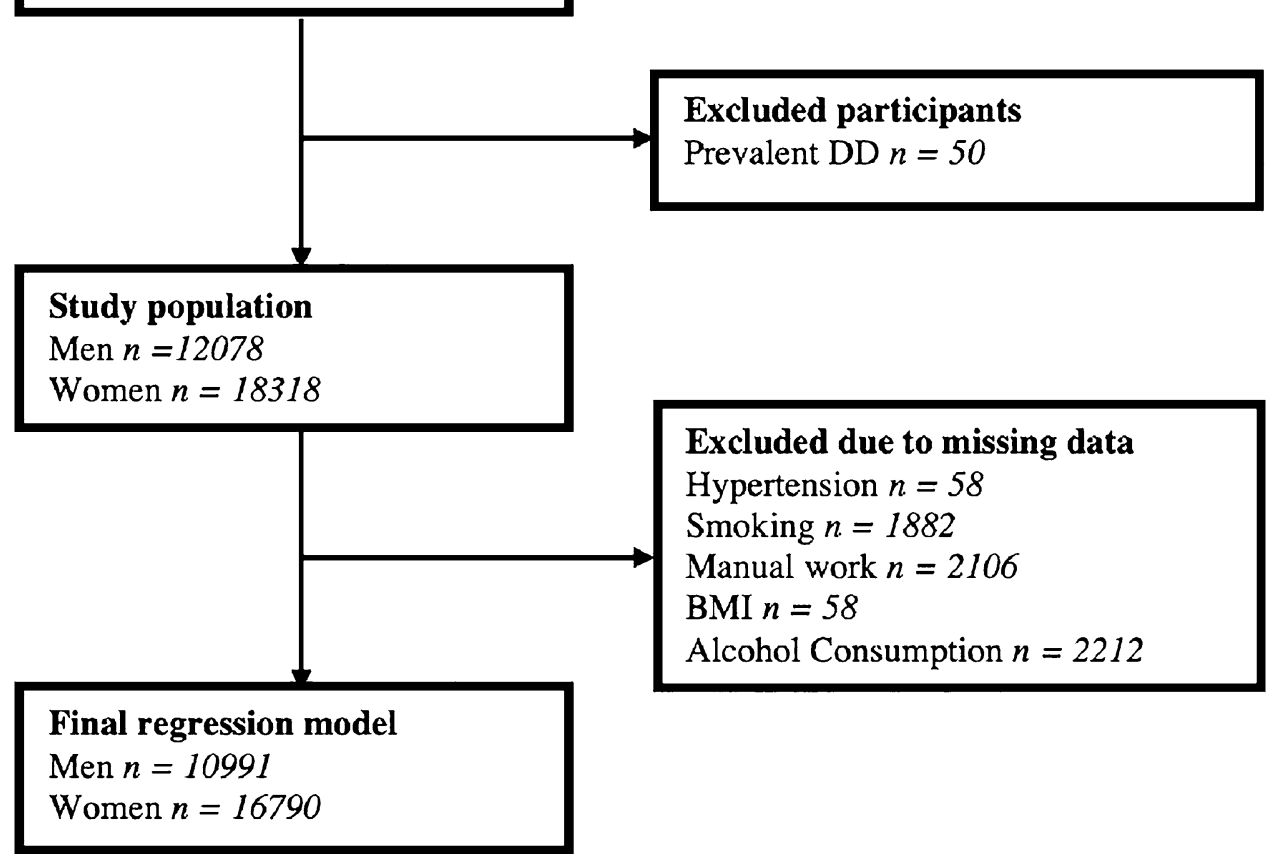

Figure 1. Derivation of the study cohort from the Malmö Diet and Cancer Study (MDCS). BMI body mass index, $D D$ Dupuytren's disease.

\begin{tabular}{|c|c|c|c|c|}
\hline & \multicolumn{2}{|l|}{ Model I* } & \multicolumn{2}{|l|}{ Model II ${ }^{* *}$} \\
\hline & HR (95\% CI) & p-value & HR (95\% CI) & p-value \\
\hline \multicolumn{5}{|l|}{ Men } \\
\hline Diabetes mellitus & $2.03(1.38-3.00)$ & $<0.001$ & $2.23(1.50-3.30)$ & $<0.001$ \\
\hline Current smoking & $1.19(0.94-1.52)$ & $=0.15$ & $1.10(0.86-1.40)$ & $=0.43$ \\
\hline Hypertension & $0.93(0.74-1.17)$ & 0.54 & $0.90(0.72-1.14)$ & $=0.38$ \\
\hline Low alcohol consumption & Reference & - & Reference & - \\
\hline Moderate alcohol consumption & $1.62(1.26-2.10)$ & $<0.001$ & $1.67(1.29-2.16)$ & $<0.001$ \\
\hline Heavy alcohol consumption & $2.37(1.79-3.14)$ & $<0.001$ & $2.46(1.85-3.27)$ & $<0.001$ \\
\hline Normal weight & Reference & - & Reference & - \\
\hline Overweight & $0.88(0.70-1.11)$ & $=0.27$ & $0.85(0.67-1.07)$ & $=0.17$ \\
\hline Obesity & $0.70(0.47-1.05)$ & $=0.08$ & $0.66(0.44-0.98)$ & $=0.04$ \\
\hline Manual work & $1.00(0.80-1.26)$ & $=0.98$ & $1.11(0.98-1.02)$ & $=0.38$ \\
\hline \multicolumn{5}{|l|}{ Women } \\
\hline Diabetes mellitus & $2.38(1.32-4.29)$ & $=0.004$ & $2.69(1.48-4.90)$ & $=0.001$ \\
\hline Current smoking & $0.94(0.67-1.32)$ & $=0.73$ & $0.86(0.61-1.22)$ & $=0.40$ \\
\hline Hypertension & $0.88(0.64-1.21)$ & $=0.43$ & $0.88(0.64-1.12)$ & $=0.43$ \\
\hline Low alcohol consumption & Reference & - & Reference & - \\
\hline Moderate alcohol consumption & $1.68(1.17-2.42)$ & $=0.005$ & $1.75(1.20-2.53)$ & $=0.003$ \\
\hline Heavy alcohol consumption & $3.35(1.85-6.07)$ & $<0.001$ & $3.56(1.95-6.50)$ & $<0.001$ \\
\hline Normal weight & Reference & - & Reference & - \\
\hline Overweight & $1.07(0.78-1.47)$ & $=0.66$ & $1.04(0.76-1.43)$ & $=0.80$ \\
\hline Obesity & $0.61(0.36-1.06)$ & $=0.08$ & $0.57(0.33-1.00)$ & $=0.05$ \\
\hline Manual work & $1.06(0.78-1.43)$ & $=0.71$ & $1.21(0.89-1.66)$ & $=0.23$ \\
\hline
\end{tabular}

Table 3. Sex-stratified Cox regression models with HR for incident DD in relation covariates. CI confidence interval, $D D$ Dupuytren's disease, DM Diabetes Mellitus, HR Hazard Ratio. Bold values indicating $p<0.05$. ${ }^{\star}$ Cox regression model including only age at study entry and respective variable. ${ }^{* \star}$ Including age, DM, hypertension, smoking, alcohol consumption group, weight group, and manual work. 


\begin{tabular}{|l|l|l|}
\hline Variable & HR $(\mathbf{9 5 \%} \mathbf{C I})^{\mathbf{a}}$ & p-value \\
\hline Men & $1.22(1.09-1.38)$ & $<\mathbf{0 . 0 1}$ \\
\hline ApoA1 & $0.97(0.86-1.09)$ & $=0.64$ \\
\hline ApoB & $0.85(0.75-0.96)$ & $=\mathbf{0 . 0 1}$ \\
\hline ApoB/ApoA1 & $1.17(1.01-1.36)$ & $=\mathbf{0 . 0 3}$ \\
\hline Women & $0.83(0.70-0.99)$ & $=\mathbf{0 . 0 3}$ \\
\hline ApoA1 & $0.80(0.66-0.97)$ & $=\mathbf{0 . 0 2}$ \\
\hline ApoB & &
\end{tabular}

Table 4. Sex-stratified, multivariable Cox regression analysis with HR for incident DD in relation to ApoA1, $\mathrm{ApoB}$ and the ApoB/ApoA1 ratio, adjusted for age at baseline, hypertension, DM, alcohol consumption, BMI, manual work, and smoking. $B M I$ body mass index, $C I$ confidence interval, $D D$ Dupuytren's disease, $H R$ Hazard Ratio. Bold values indicating $p<0.05$. ${ }^{a} \mathrm{HR}$ are expressed as per one SD increase of respective Z-score converted variable.

Alcohol consumption and manual work. In our study, excessive alcohol consumers of both sexes had a marked higher risk of developing DD during follow-up, and these results are in line with several previous studies linking alcohol overconsumption to the development of $\mathrm{DD}^{5,6}$. Alcohol guidelines and thresholds for risk consumption differ notably from country to country ${ }^{28}$, but our study shows that already a somewhat moderate alcohol consumption (1-2 standard drinks per day), well below the definition of low-risk consumption in some countries $^{29}$, is associated with an increased risk of DD. By adding long term follow-up data, our study strengthens the previously reported associations, establishing even a relatively low alcohol overconsumption as a major risk factor for developing DD. Furthermore, several previous studies have proposed manual work as a risk factor for the development of $\mathrm{DD}^{8}$, possibly through repeated microtrauma to the hand ${ }^{30}$. We found, however, no such associations in our study, neither among men or women, possibly due to the low number of farmers in the study population.

BMI and apolipoproteins. The epidemiological literature on circulating lipids, apolipoproteins and risk of DD is scarce, and the most cited papers date back to the 1980 's and $1990^{\prime 14,15}$. In recent years, however, an inverse association between a high BMI and the risk of DD have been reported in several studies ${ }^{11,12,31}$, indicating a possible protective factor linked to the fat tissue. The putative biological mechanisms behind this associations is not known but lower serum testosterone among obese individuals has been proposed as one hormonal factor of importance, possibly decreasing the risk for $\mathrm{DD}^{31}$. In our study, we found a similar pattern of associations; men with incident DD during follow-up had a lower mean BMI at baseline compared to men without DD. Additionally, obese men had lower risk of DD compared to men with normal weight in the adjusted regression model, although the same results could not be convincingly shown among women.

However, we did find a strong association among both men and women between apolipoprotein levels and incident DD. Participants with a high ApoB/ApoA1 ratio had a lower risk of DD even when adjusting for age, $\mathrm{DM}$, alcohol consumption, and BMI. Traditionally, the ApoB/ApoA1 ratio has been used as a predictor of cardiovascular disease $\mathrm{e}^{32,33}$ and analysis of ApoB is still recommended as a complement or alternative to LDL-C in risk assessment of cardiovascular disease ${ }^{34}$. Our study is to the best of our knowledge the first to analyze apolipoprotein levels in relation to DD, why further studies are warranted, firstly to confirm these results, and secondly to better understand the biochemical background to the reported associations. Interestingly, recent genetic studies have proposed a negative genetic correlation between high BMI and $\mathrm{DD}^{13}$, supporting the previous results associating lower BMI with $\mathrm{DD}^{11,12}$. Furthermore, in studies on over 30,000 Danish twin pairs, the hereditability of DD was approximately $80 \%$, whereas environmental risk factors, e.g. smoking or alcohol consumption, only contributes to $20 \%$ of the increased risk $^{9}$. A recent Swiss study found that family history of DD was a strong risk factor for incident $\mathrm{DD}$, emphasising the importance of genetic susceptibility for the development of the disease ${ }^{35}$. This could indicate that the associations found between apolipoproteins, low BMI and DD are due to genetic correlation rather than fat tissue being protective against DD. Since no genetic information was available in this study, we cannot draw any conclusions regarding such associations from our results, but it is an obvious target for future studies. Another future research target would be to examine palmar fascia biopsies from overweight and obese patients with and without DD, aiming to better understand the interplay between BMI, fat tissue, and DD risk.

To summarize, there might exist different protective and predisposing factors for the development of DD. Genetic predisposition, hormonal factors related to sex or high BMI, alterations in the palmar fascia due to DM and hyperglycemia, and finally, environmental factors such as alcohol consumption all interact in the complex pathogenesis behind DD (Supplementary Fig. S1).

Strengths and limitations. One of the limitations of this study is the lack of standardized diagnostic criteria for DD during follow-up. The diagnoses were retrieved using ICD-codes and thus, physicians might have applied different criteria for diagnosis. Since we only used diagnoses from a specialized care setting, however, most diagnoses were established by trained specialists in hand or orthopedic surgery, strengthening case valid- 
ity. Some participants might have been diagnosed with DD in primary care without specialist referral, which might have lowered our incidence rates to some extent. Indeed, data on incidence rates in our study are slightly lower than in a previous study from southern Sweden including data from primary care ${ }^{4}$, indicating that not all patients in Sweden with DD are referred to specialized care. Furthermore, since DD is a benign disease, some participants with moderate symptoms might not have sought healthcare at all despite having the disease and were thus not included in our registers. Taken together, our study design allowed us to analyze a clinically relevant diagnosis of DD, established in the specialized care setting, why results should be interpreted with this in mind. Another strength of this longitudinal study design is the lack of recall bias among participants, something that may otherwise be problematic in case-control studies.

Furthermore, a major strength of this study is the large population size, over 30,000 middle-aged individuals followed over 20 years making this the largest cohort study to date analyzing risk factors for DD. Moreover, the collection of baseline data was of high quality, with each participant being examined individually by a trained nurse. The data regarding prevalent DM are also of high quality, using several local and national registers for diagnosis acquirement. Unfortunately, we were not able to reliably stratify for type 1 and type 2 DM, making separate analyses for different types of $\mathrm{DM}$ a target for future research. Levels of glycemic control $\left(\mathrm{HbA}_{1 \mathrm{c}}\right)$ in relation to DD risk would also be an interesting topic for research. Finally, a participant's baseline characteristics, such as BMI and alcohol consumption, were only measured once. Undoubtably, these variables often change over a person's lifetime, and this is a limitation that should be kept in mind when interpreting our results.

Even so, despite the high quality of baseline data and our adjustments for known confounders in the regression models, there is always a possibility that additional confounders might have influenced our results. With all this in mind, our conclusions are in line with several previous studies indicating both DM and excessive alcohol consumption as risk factors for the development of DD, thus making it unlikely that residual confounding would be sufficient to alter our main results significantly, but caution should always be taken when interpreting data from observational studies.

\section{Conclusion}

DM and excess alcohol consumption constituted major risk factors for the development of DD in middle-aged subjects irrespective of sex. This study adds information regarding the debated pathogenesis underlying DD, but additional studies are warranted to further elucidate potential genetic markers for associations between DD, BMI and apolipoproteins.

\section{Methods}

Study population. A cohort from the $\mathrm{MDCS}^{17}$, consisting of 30,446 individuals from southern Sweden was studied. The participants living in the city of Malmö, born between 1926 and 1945 were invited to participate in the study between 1991 and 1996. Subjects underwent clinical examination, laboratory assessment and filled in a questionnaire regarding cardiovascular risk factors. The recruitment process has been previously described in detail $^{17}$, the attendance rate was approximately $41 \%$, and $60 \%$ of the participants were women.

Baseline data collection. At baseline, a trained nurse measured participant's height and weight. BMI was calculated as a participant's weight $/ \mathrm{length}^{2}\left(\mathrm{~kg} / \mathrm{m}^{2}\right)$ and divided into three categories; $<25$ (normal weight), $\geq 25$ to $<30$ (overweight), and $\geq 30$ (obese). Blood pressure (BP) was measured in a supine position with a mercury column sphygmomanometer, and hypertension was defined as a systolic BP $\geq 140 \mathrm{mmHg}$ or diastolic $\mathrm{BP} \geq 90 \mathrm{mmHg}$. Smoking was self-reported at baseline and defined as current smoker or non-smoker. Alcohol consumption was also self-reported as consumption during the last week in grams per day and divided into low consumers (<15 g/day), moderate consumers (15-30 g/day) and heavy consumers ( $>30 \mathrm{~g} /$ day). DM at baseline was defined as a fasting whole blood glucose $>7.0 \mathrm{mmol} / \mathrm{L}$, a self-reported physician's diagnosis or the use of antidiabetic medicine. Data on prevalent DM was also obtained using six other national and local registers as previously described in detail ${ }^{36}$. Data on current or latest occupation was based on self-reported job titles and categorized according to Swedish national population census ${ }^{37}$. Participants were classified as manual workers, which also included farming, and non-manual workers as previously described ${ }^{38}$.

At baseline, blood samples were collected from the participants and stored at $-80{ }^{\circ} \mathrm{C}$. Levels of ApolipoproteinA1 (ApoA1) and Apolipoprotein B (ApoB) in serum were analysed by Quest Diagnostics (San Juan Capistrano, CA, USA) using an immunonephelometric assay run on a Siemens BNII (Siemens, Newark, DE, USA) in a total of 27,753 participants.

Endpoints. Using Swedish national registers (Cause of Death Register, the Inpatient and Outpatient Registers) administrated by the National Board of Health and Welfare (http://socialstyrelsen.se/english), primary endpoint diagnosis, i.e. DD, were obtained by linkage of registers using each participants unique 10-digit personal number ${ }^{39}$. Using the International Classification of Disease (ICD) Swedish version 8, 9 or 10, both prevalent and incident diagnoses of DD were retrieved with the following ICD codes: 733.90/728G/M720. Only clinical, hospital-based diagnoses were used in this study, operation codes were not included. As the registers did not include diagnoses from primary health care, the study only includes diagnoses established in the specialized care mainly by specialists in hand- or orthopaedic surgery. Participants were followed from baseline until a diagnosis of DD in either of the registers, emigration, death, or end of study 2018-12-31, thus creating a time-to-event variable unique for each participant. Participants with a diagnosis of DD prior to baseline examination were excluded from further study. 
Statistical analysis. Baseline quantitative data were presented as mean with standard deviation (SD) when normally distributed and as median with interquartile range [IQR] when data was skewed. Count and proportion were used for presentation of nominal data. For group comparisons, independent sample t-test was used for age, BMI, ApoA1 and ApoB levels, Chi-Square test for dichotomous variables and Mann-Whitney $U$ test was used for alcohol consumption. Levels of ApoA1, ApoB and the ApoB/ApoA1 ratio were analysed after a $\mathrm{z}$-score standardization ([Variable level - mean]/standard deviation) resulting in a $\mathrm{Z}$-score variable with a mean of 0 and a SD of 1 .

Potential associations between DD and prevalent DM, apolipoprotein levels, BMI, manual work and alcohol consumption were analyzed in Cox proportional hazards models stratified by sex. Two models were used; the first model included only age and the respective variable. The second multivariable model included BMI group, smoking habits, alcohol consumption, prevalent diabetes at baseline, hypertension, and manual work; i.e. the studied variable and possible confounders ${ }^{5,7,10,11}$. Hazard ratios (HR) with $95 \%$ confidence intervals (CI) were expressed with participants without DM at baseline as a reference when analysing DM, BMI $<25 \mathrm{~kg} / \mathrm{m}^{2}$ as reference when analysing BMI groups, and low alcohol consumers as reference when analysing alcohol consumption. For ApoA1, ApoB and ApoB/ApoA1 ratio, HR with 95\% CI were expressed as per one SD increment of each $\mathrm{z}$-score converted variable.

To test for potential interaction between DM, alcohol consumption, and BMI, interaction variables $(\mathrm{DM} \times \mathrm{Alcohol})$ and $(\mathrm{DM} \times \mathrm{BMI})$ were introduced on top of the sex stratified, age-adjusted Cox regression models and analyzed, using continuous variables for alcohol consumption and BMI.

Finally, two sensitivity analyses were performed. The first one excluded participants with $\mathrm{BMI}<20$ in the multivariable Cox regression model, and the second one excluded participants with a follow-up time $\geq 25$ years.

The assumption of proportional hazard was assessed by visual assessment of Kaplan-Meier curves and log-log plots and no violation was found. The statistical analyses were conducted using SPSS for Mac version 25 (SPSS Inc., Chicago, IL, USA). A two-tailed $p$ value $<0.05$ was considered significant.

Ethics approval. The participants provided informed consent and both the MDCS and the present study were approved by the ethical committee at Lund University (DNR: LU51-90; 2009-633; 2019-01439). The study was conducted in accordance with the Helsinki Declaration.

\section{Data availability}

The data used for this study can be applied for by contacting the Steering Committee of the Malmö Diet and Cancer Study; (data manager Anders Dahlin, email: anders.dahlin@med.lu.se). For details of MDCS and how to apply for data, see web site: www.malmo-kohorter.lu.se/english.

Received: 9 December 2020; Accepted: 30 June 2021

Published online: 19 July 2021

\section{References}

1. Dutta, A. et al. Dupuytren's contracture-Current concepts. J. Clin. Orthop. Trauma 11, 590-596 (2020).

2. Lanting, R., Broekstra, D. C., Werker, P. M. \& van den Heuvel, E. R. A systematic review and meta-analysis on the prevalence of Dupuytren disease in the general population of Western countries. Plast. Reconstr. Surg. 133, 593-603 (2014).

3. Hindocha, S., McGrouther, D. A. \& Bayat, A. Epidemiological evaluation of Dupuytren's disease incidence and prevalence rates in relation to etiology. Hand (N. Y.) 4, 256-269 (2009).

4. Nordenskjold, J., Englund, M., Zhou, C. \& Atroshi, I. Prevalence and incidence of doctor-diagnosed Dupuytren's disease: A population-based study. J. Hand Surg. Eur. 42, 673-677 (2017).

5. Godtfredsen, N. S., Lucht, H., Prescott, E., Sørensen, T. I. \& Grønbaek, M. A prospective study linked both alcohol and tobacco to Dupuytren's disease. J. Clin. Epidemiol. 57, 858-863 (2004).

6. Descatha, A. et al. Association among work exposure, alcohol intake, smoking and Dupuytren's disease in a large cohort study (GAZEL). BMJ Open 4, e004214 (2014).

7. Wijnen, V., Buntinx, F., De Smet, L., Bartholomeeusen, S. \& Degreef, I. Comorbidity in Dupuytren disease. Acta Orthop. Belg. 82, 643-648 (2016).

8. Mikkelsen, O. A. Dupuytren's disease-The influence of occupation and previous hand injuries. Hand 10, 1-8 (1978).

9. Larsen, S. et al. Genetic and environmental influences in Dupuytren's disease: A study of 30,330 Danish twin pairs. J. Hand Surg. (Eur. Vol.) 40, 171-176 (2014).

10. Broekstra, D. C., Groen, H., Molenkamp, S., Werker, P. M. N. \& van den Heuvel, E. R. A systematic review and meta-analysis on the strength and consistency of the associations between Dupuytren disease and diabetes mellitus, liver disease, and epilepsy. Plast. Reconstr. Surg. 141, 367e-379e (2018).

11. Hacquebord, J. H., Chiu, V. Y. \& Harness, N. G. The risk of Dupuytren diagnosis in obese individuals. J. Hand Surg. 42, 149-155 (2017).

12. Gudmundsson, K. G., Arngrmsson, R., Sigfússon, N., Björnsson, Á. \& Jónsson, T. Epidemiology of Dupuytren’s disease: Clinical, serological, and social assessment. The Reykjavik Study. J. Clin. Epidemiol. 53, 291-296 (2000).

13. Major, M. et al. Integrative analysis of Dupuytren's disease identifies novel risk locus and reveals a shared genetic etiology with BMI. Genet. Epidemiol. 43, 629-645 (2019).

14. Sanderson, P. L., Morris, M. A., Stanley, J. K. \& Fahmy, N. R. Lipids and Dupuytren's disease. J. Bone Jt. Surg. Br. 74, $923-927$ (1992).

15. Rabinowitz, J. L., Ostermann, L., Bora, F. W. \& Staeffen, J. Lipid composition and de novo lipid biosynthesis of human palmar fat in Dupuytren's disease. Lipids 18, 371-374 (1983).

16. Hacquebord, J. H. \& Harness, N. G. The relationship of normal BMI and Dupuytren's disease. Minerva Ortopedica e Traumatologica 68, 216-219 (2017).

17. Berglund, G., Elmstahl, S., Janzon, L. \& Larsson, S. A. The Malmo diet and cancer study. Design and feasibility. J. Intern. Med. 233, 45-51 (1993).

18. Goh, S. Y. \& Cooper, M. E. Clinical review: The role of advanced glycation end products in progression and complications of diabetes. J. Clin. Endocrinol. Metab. 93, 1143-1152 (2008). 
19. Bodiga, V. L., Eda, S. R. \& Bodiga, S. Advanced glycation end products: Role in pathology of diabetic cardiomyopathy. Heart Fail. Rev. 19, 49-63 (2014).

20. Kyung, S. Y. et al. Advanced glycation end-products and receptor for advanced glycation end-products expression in patients with idiopathic pulmonary fibrosis and NSIP. Int. J. Clin. Exp. Pathol. 7, 221-228 (2013).

21. Sessions, J. \& Nickerson, D. S. Biologic basis of nerve decompression surgery for focal entrapments in diabetic peripheral neuropathy. J. Diabetes Sci. Technol. 8, 412-418 (2014).

22. Takase, F. et al. Association of advanced glycation end products in Dupuytren disease. J. Orthop Surg. Res. 13, 143 (2018)

23. Yuen, A. et al. Methylglyoxal-modified collagen promotes myofibroblast differentiation. Matrix Biol. 29, 537-548 (2010).

24. Verhoekx, J. S. N. et al. Isometric contraction of Dupuytren's myofibroblasts is inhibited by blocking intercellular junctions. J. Investig. Dermatol. 133, 2664-2671 (2013).

25. Papanas, N. \& Maltezos, E. The diabetic hand: A forgotten complication?. J. Diabetes Complicat. 24, 154-162 (2010).

26. Rydberg, M. et al. Diabetes mellitus as a risk factor for compression neuropathy: A longitudinal cohort study from southern Sweden. BMJ Open Diabetes Res. Care 8, e001298 (2020).

27. Zimmerman, M. et al. Ulnar nerve entrapment in diabetes: Patient-reported Outcome after Surgery in National Quality Registries. Plast. Reconstr. Surg. Glob. Open 8, e2740 (2020).

28. Wood, A. M. et al. Risk thresholds for alcohol consumption: Combined analysis of individual-participant data for 599912 current drinkers in 83 prospective studies. Lancet 391, 1513-1523 (2018).

29. Kalinowski, A. \& Humphreys, K. Governmental standard drink definitions and low-risk alcohol consumption guidelines in 37 countries. Addiction 111, 1293-1298 (2016).

30. Descatha, A. et al. Heavy manual work, exposure to vibration and Dupuytren's disease? Results of a surveillance program for musculoskeletal disorders. Occup. Environ. Med. 69, 296-299 (2012).

31. Majeed, M., Wiberg, A., Ng, M., Holmes, M. V. \& Furniss, D. The relationship between body mass index and the risk of development of Dupuytren's disease: a Mendelian randomization study. J. Hand Surg. (Eur. Vol.) 46, 406-410 (2020).

32. Walldius, G. \& Jungner, I. The apoB/apoA-I ratio: A strong, new risk factor for cardiovascular disease and a target for lipid-lowering therapy-A review of the evidence. J. Intern. Med. 259, 493-519 (2006).

33. Schmidt, C. \& Bergstrom, G. Apolipoprotein B and apolipopotein A-I in vascular risk prediction-A review. Curr. Pharm. Des. 20, 6289-6298 (2014).

34. Mach, F. et al. 2019 ESC/EAS Guidelines for the management of dyslipidaemias: Lipid modification to reduce cardiovascular risk: The Task Force for the management of dyslipidaemias of the European Society of Cardiology (ESC) and European Atherosclerosis Society (EAS). Eur. Heart J. 41, 111-188 (2020).

35. Becker, K. et al. The importance of genetic susceptibility in Dupuytren's disease. Clin. Genet. 87, 483-487 (2015).

36. Enhörning, S., Hedblad, B., Nilsson, P. M., Engström, G. \& Melander, O. Copeptin is an independent predictor of diabetic heart disease and death. Am. Heart J. 169, 549-556.e541 (2015).

37. Statistics_Sweden. Occupations in Population and Housing Census 1985 (FoB 85) According to Nordic Standard Occupational Classification (Nordisk yrkesklassificering NYK) and Swedish Socio-economic Classification (Socioekonomisk indelning SEI) (Statistics Sweden, 1989).

38. Manjer, J., Elmståhl, S., Janzon, L. \& Berglund, G. Invitation to a population-based cohort study: Differences between subjects recruited using various strategies. Scand. J. Public Health 30, 103-112 (2002).

39. Ludvigsson, J. F. et al. External review and validation of the Swedish national inpatient register. BMC Public Health 11, 450-450 (2011).

\section{Author contributions}

A significant contribution to this manuscript was made by the authors. The study was designed by M.R., M.Z. and L.D., the first draft was made by M.R. and all authors contributed with intellectual content and to the data interpretation. Before publishing, the stated authors reviewed and accepted the final version.

\section{Funding}

Open access funding provided by Lund University. The Malmö Diet and Cancer study was funded by grants from the Swedish Cancer Foundation, the Swedish Medical Research Council, AFA insurance, the Albert Påhlsson and Gunnar Nilsson Foundations and the Malmö city council. This study was funded by Swedish Diabetes Foundation, the Regional Agreement on Medical Training and Clinical Research (ALF) between Region Skåne and Lund University, Stig and Ragna Gorthons foundation.

\section{Competing interests}

The authors declare no competing interests.

\section{Additional information}

Supplementary Information The online version contains supplementary material available at https://doi.org/ 10.1038/s41598-021-94025-7.

Correspondence and requests for materials should be addressed to M.R.

Reprints and permissions information is available at www.nature.com/reprints.

Publisher's note Springer Nature remains neutral with regard to jurisdictional claims in published maps and institutional affiliations. 
(c) (i) Open Access This article is licensed under a Creative Commons Attribution 4.0 International cc) License, which permits use, sharing, adaptation, distribution and reproduction in any medium or format, as long as you give appropriate credit to the original author(s) and the source, provide a link to the Creative Commons licence, and indicate if changes were made. The images or other third party material in this article are included in the article's Creative Commons licence, unless indicated otherwise in a credit line to the material. If material is not included in the article's Creative Commons licence and your intended use is not permitted by statutory regulation or exceeds the permitted use, you will need to obtain permission directly from the copyright holder. To view a copy of this licence, visit http://creativecommons.org/licenses/by/4.0/.

(C) The Author(s) 2021 